\title{
EDUCATION AND TRAINING The great escape? The rise of the escape room in medical education
}

\author{
Authors: Jonathan Guckian, ${ }^{A}$ Leanne Eveson ${ }^{B}$ and Hannah May ${ }^{C}$
}

Medical education has changed focus to a more learner-centred model, placing learners at the centre of innovations in training. The escape room is one such innovative learner-focused activity, in which a team of players cooperatively discover clues, solve puzzles and complete tasks in order to progress through the challenge to achieve a specific goal. Escape rooms can be used in medical education as a tool for team building, an entertaining way of delivering technical and non-technical skills, to read and acquire or refresh knowledge, as well as for educational research. Despite appearing to be a superficial form of entertainment, escape rooms can be grounded in sound educational theory and, when used effectively, act as a low-cost, high-impact resource for a variety of learners. While escape rooms may well be an example of yet another educational 'fad' demonstrating the rising influence of 'Millennial MedEd', it signals a promising shift to more learner-centred, team-based methods which are essential to the practice of safe modern healthcare during the current COVID-19 pandemic and beyond.

KEYWORDS: Medical education, human factors, team building, gamification, escape rooms

DOI: 10.7861/fhj.2020-0032

\section{Background}

Medical education is characterised by rapid innovation and evolution, with profound shifts to more learner-centred activities over the past 2 decades, in parallel with patient-centred care placing the needs of learners at the focal point of educational delivery. ${ }^{1}$ We detail the rise of the escape room as one such learner-centred learning activity.

Authors: ${ }^{\text {A }}$ core medical trainee, Leeds Teaching Hospitals NHS Trust, Leeds, UK, Director for Communications \& Social Media, Association for the Study of Medical Education, Edinburgh, UK and Founder, Medisense Medical Education, Leeds UK; ${ }^{\mathrm{B}}$ cardiology registrar, Frimley Health NHS Foundation Trust, Frimley Park, UK and Royal Air Force Medical Service, UK; ' ${ }^{\text {S }}$ senior clinical fellow in diabetes and endocrinology, University Hospital Lewisham, Lewisham, UK and education and leadership fellow, Health Education England, London, UK
The original escape rooms were conceived as a game, in which a team of players (typically between two and 10 team members) cooperatively discover clues, solve puzzles and complete tasks in one or more rooms in order to progress and achieve a specific goal. This format was popularised in the TV series The Crystal Maze and Fort Boyard in the 1990s, and has now been brought into the realm of mass public entertainment with the explosion of gaming as a social activity. Games are set in a variety of fictional settings, for example a doctor's surgery, a prison cell or a space station. Rules are set out at the beginning of the session with details of how to win and 'escape'. If players achieve the goal within the time limit, they win the game. Hints can be provided to assist the team when necessary and a debrief is carried out afterwards.

There are now estimated to be over 50,000 escape rooms worldwide and the UK has seen the market grow rapidly over the past 5 years. ${ }^{2}$ Escape room games are becoming embedded in society, from widespread popularity for small group entertainment to use in the home, for example via Amazon Alexa software.

In parallel, there has been increased use in medical education, as an adjunct to traditional teaching methods. We aim to briefly outline the methods of use of escape rooms in medical education, attempt to explain their popularity and critically assess their potential advantages, referring to educational theory.

\section{Escape rooms and medical education}

Escape rooms can be used in medical education as a tool for team building, a fun way of delivering learning material, human factors and have also been used in healthcare recruitment. ${ }^{3-5}$ The steps required for development of a medical escape room, as well as examples of their uses, are detailed below.

\section{Case study 1: Escape rooms as a tool for team building}

To develop teamwork and cohesiveness, 15 core medical trainees (CMTs) and two members of a multidisciplinary team from two London teaching hospitals took part in 'The escape room challenge'. Outcome parameters were assessed pre- and postescape room using a questionnaire.

All four teams successfully completed the challenges. All participants enjoyed the session and felt that a dedicated teambuilding session for CMTs and multidisciplinary team members was beneficial. Specific identified benefits included an opportunity to learn personal strengths and weaknesses, capabilities of peers 
in a non-clinical setting and to see how individuals respond in time-pressured and stressful environments.

This case study demonstrated that escape rooms can enhance communication, increase awareness of responsibility and improve morale for CMT doctors without significant costs.

\section{Case study 2: Escape rooms as a tool for research}

One study on escape rooms focused on providing an adjunct to traditional lecture teaching on dermatology content for clinical stage medical students. ${ }^{3}$ It had concurrent aims of demonstrating the variety of dermatological conditions on a curriculum, as well as acting as a research tool to assess student perceptions regarding dermatology as a specialty. Puzzles included spotting methotrexate prescribing errors, completing dermatology crosswords and matching pictures to suitable rash descriptors.

In order to assess its impact, the escape room was accompanied by a preceding lecture and followed by a comprehensive debrief. From a research perspective, the escape room acted as the study intervention, after which qualitative data was sought from a focus group. This is just one example of how escape rooms can fulfil multiple academic and educational purposes, including highlighting career opportunities, offering learner-centred education and advancing scholarship. This particular initiative was used as a research intervention to stimulate discussion in a focus group on prejudices in dermatology education, however, it could also be used as part of an evaluation of a learning programme. There may also be capacity for observation of team dynamics in an ethnographic context, or as an intervention in human factors or quality improvement research.

\section{Case study 3: Escape rooms as a tool for learning}

Obstetrics is a high-pressure clinical specialty. Rapid recall of clinical process, along with effective teamworking and clear communication, in time-pressured environments is often required. A clinical escape room was developed to test these skills in a controlled environment.

Trainees from specialty training year 1 (ST1) to ST7 were briefed that two patients, one with a ruptured ectopic pregnancy and another requiring a forceps delivery, needed their immediate attention. Trainees then had to solve clues to work through all the processes required to perform both laparoscopic surgery and an instrumental delivery safely. Clues were distributed in such a way that even if teams divided the cases, they would need to communicate with one another to gain all the relevant information.

Participants in the escape room felt the experience helped them learn lessons that they could apply in real-life practice, particularly around consistent communication, assignment of roles and effective leadership.

\section{Why are they popular?}

The popularity of escape rooms in education may be attributed to three factors: the learner population, the societal impact of gamification and delivery convenience.

\section{Millennial MedEd}

Much has been made of the changes in learning experience and expectations of millennial learners. Millennials, defined as individuals born between 1981 and 1996, make up the majority of the current postgraduate medical education cohort. They are a generation who grew up with technology and mass media, with easy access to personal computers and mobile phones. ${ }^{6}$ This exposure is said to have created a group who are characterised as technologically adept, goal oriented and socially confident. Millennials are described as valuing choice and variety and having a preference for collaborative, team working. ${ }^{6}$

\section{Gamification}

Gamification, or the use of game design elements in non-game contexts, brings together many of the elements that are naturally appealing to the millennial generation. Gamification has been shown to motivate learners, increase engagement and encourage social interaction in a learning environment, so it comes as no surprise that this form of educational delivery has become increasingly popular in medical education in recent years. ${ }^{7}$

Medical escape rooms provide the perfect opportunity to deliver teaching of knowledge, skills and behaviours in a gamified context. They provide educational opportunities that emphasise collaborative learning, with activities that require teamwork and clear goals, and have a built-in opportunity for rapid feedback.

Modern medical curricula now place greater emphasis on human factors. ${ }^{4}$ A traditional didactic teaching model offers limited opportunity to learn about how human factors relate to patient safety in clinical environments in a meaningful way. Escape rooms can be used as a form of medical simulation, providing a way to familiarise individuals with processes used in healthcare, as well as recreating specific experiences vital for learning in a controlled manner, with an opportunity for debrief.

\section{Convenience of delivery}

From a practical perspective, escape rooms also have a lot to offer In a busy and time-pressured NHS, they offer a discrete learning experience that can be completed in less than an hour. While their initial design requires a significant time investment, they can be reused for subsequent groups with little or no adaptation. This creative, non-patient-facing format can provide high-quality medical education to small groups without sacrificing significant clinical time. As more 'disruptive' educational tools are being developed in a field ravaged by the COVID-19 pandemic, escape rooms may provide an alternative means of team-building or medical student curriculum delivery, and crucially do not require patient contact. Provided that clear instructions are left by the designer, they do not require an expert facilitator, unlike the delivery of a lecture or seminar. Finally, most escape room set-ups are low cost, with a budget of around $£ 50$ normally covering all materials required.

\section{How may educational theory provide understanding of learning using escape rooms?}

Popularity may add some weight to the escape room as a learning tool, however as with any medical education intervention, it is important to cast a critical eye on this apparent 'easy win'. The most sustainable educational innovations are underpinned by theoretical frameworks. One may ask, therefore, how does educational theory apply to the escape room? 

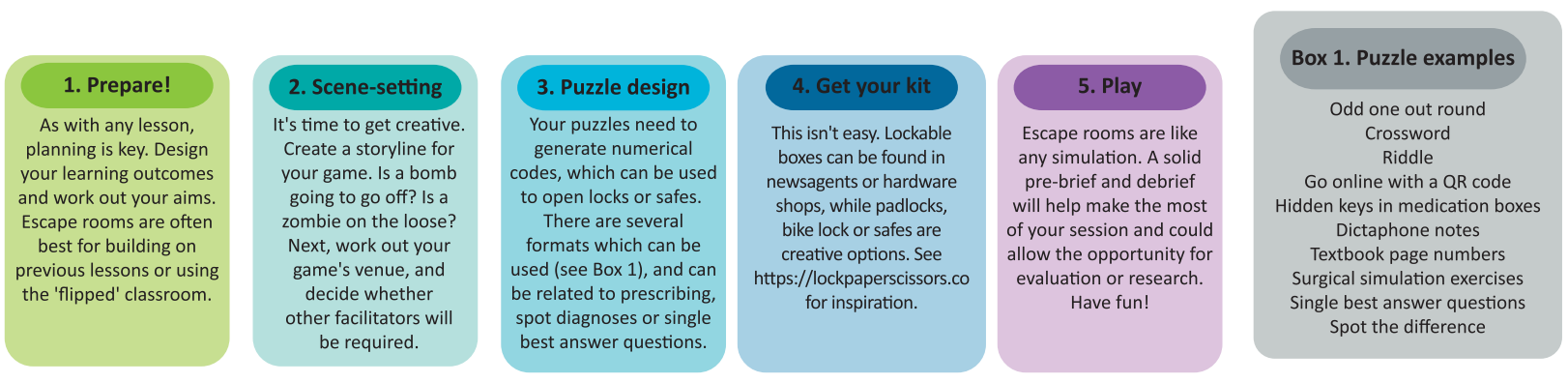

Fig 1. A guide to developing a medical escape room.

One answer lies in ease of use for a variety of learning needs. As with the case studies featured in this article, the escape room can be a flexible tool for the educationalist, used for a multitude of purposes, from team building (case study 1) to tools for evaluation of educational practice (case study 2).

Escape rooms are effectively being used as a method of simulation-based education (SBE). Simulation is a now wellestablished educational practice, which is generally highly valued by learners. It can be mapped effectively to Kolb's experiential learning cycle, as it permits active experimentation in a safe environment, prior to undertaking concrete experiences 'in the wild'. ${ }^{8}$ Debrief and reflection are essential to learning in Kolb's cycle, and the real value of the escape room could be argued to be purely these elements, in common with other types of SBE. Considering escape rooms as a type of SBE likely extends to the other benefits in teaching human factors and other patient safety principles. Experiential learning methods are particularly popular in patient safety education due to the need for a risk-free environment; therefore it is unsurprising that the escape room has been highlighted in patient safety as a next step beyond simple simulation. ${ }^{4}$

A primary difference between traditional simulation methods and the escape room is in its 'gamified' nature. While published theoretical concepts on the use of gamification in medical education are still evolving, self-determination theory is regarded as a robust model for considering learning within this paradigm. Self-determination is a motivation theory, with the concept of a spectrum of drive to succeed, influenced by extrinsic factors (ie reward or punishment) and intrinsic factors (ie personal enjoyment). ${ }^{9}$ Self-determination theory highlights the importance of fulfilment of psychosocial needs of competence, relatedness (belonging) and autonomy. A good escape room, therefore, sets achievable goals for participants (competence), facilitates freedom of choice for learners (autonomy) and features effective teamwork and facilitation (relatedness). Some gamification literature suggests that peer-to-peer activity can stimulate motivation and subsequent learning. ${ }^{7}$ Much of the current evidence in the escape room literature features peer teambuilding activities and near-peer facilitation. The flattening of traditional hierarchies may be a motivational driver for learning in the escape room.

Finally, stepping back from the activities involved in the escape room, one could consider its place in curricular delivery. Traditional scholarly academic curricula are content-focused and essentially ignore personal development. Medical schools have begun to move to more learner-centred models, which in recent years have been built on self-directed learning and the flipped classroom. Escape rooms have been described as an example of the recent proliferation of such learner-centred activities. ${ }^{3}$

Although use of the escape room has risen in prominence, its sustainability could be called into question. There is a plethora of evidence of trendy 'gamechangers' in medical education, which have varied greatly in their shelf-life. Problem-based learning, for example, once so highly regarded that it became the primary delivery tool of some curricula, has lost favour, albeit still in use in some medical schools to a more limited degree. There are also numerous examples of less sustainable interventions, particularly in technology enhanced learning (TEL), in which social media-based platforms rise to worldwide prominence before disappearing, leaving only cautionary tales of lost educational content. ${ }^{10}$ Educators should be vigilant of such trends and be mindful that important lessons can be learned regardless of the sustainability of any new intervention. It remains to be seen whether the escape room, grounded in theory or not, will just be another popular educational intervention, valued by students and educators for a time before fading away. In an age disrupted by COVID-19, however, the development of virtual escape rooms may provide some promise for those seeking educational tools compatible with social distancing. Much like online gaming, resources such as the Alexa software can be accessed on-demand for individuals or groups. These advances could enhance flipped classroom learning and their on-demand nature is suited to a learner-centred paradigm. It is likely that technology-enhanced learning initiatives will become more prominent as the medical education landscape is reorganised following COVID-19. Escape rooms may therefore be considered as an option to replace or augment traditional learning no longer deliverable in person.

\section{Conclusion}

Escape rooms have potential as a novel role of delivery in educational practice, with potential uses in team building, enhancing traditional approaches to simulation and providing a medium for less conventional learning activities. Despite potentially being perceived as a superficial form of entertainment, escape rooms can be grounded in modern educational theory and, when used effectively, can act as low-cost, high-impact resource for a variety of learners. While the escape room is just one example of gamification in medical education, it does support the important shift to more learner-centred curricula and demonstrates the rising influence of 'Millennial MedEd'. Escape rooms can be practically challenging to run but have potential for 
high pay-off when used correctly. While they are currently popular, the jury remains out on their efficacy. The need for convenience education in the current response of healthcare to the COVID-19 pandemic provides an ideal environment in which to test efficacy at pace - we look forward to learning more.

\section{References}

1 Spencer JA, Jordan RK. Learner centred approaches in medical education. BMJ 1999;318:1280-3.

2 The Logic Escapes Me. International Escape Room Markets analysis. The Logic Escapes Me, 2019. https://thelogicescapesme.com/ news/international-escape-room-markets-analysis [Accessed 21 February 2020].

3 Guckian J, Sridhar A, Meggitt SJ. Exploring the perspectives of dermatology undergraduates with an escape room game. Clin Exp Dermatol 2020;45:153-8.

4 Backhouse A, Malik M. Escape into patient safety: bringing human factors to life for medical students. BMJ Open Quality 2019;8:e000548

5 Wiemker M, Elumir E, Clare A. Escape room games: Can you transform an unpleasant situation into a pleasant one? In Haag ], Weißenböck J, Gruber MW, Christian M, Freisleben-Teutscher F (eds), Game based learning. St. Pölten: Fachhochschule St Pölten, 2015:55-68.

6 Wilson M, Gerber L. How generational theory can improve teaching: strategies for working with the "millennials". Curr Teach Learn 2007:1:29-39.

7 Rutledge C, Walsh C, Swinger N et al. Gamification in action. Academic Medicine 2018;93:1014-20.

8 Poore J, Cullen D, Schaar G. Simulation-based interprofessional education guided by Kolb's experiential learning theory. Clinical Simulation in Nursing 2014;10:e241-7.

9 Ten Cate O, Kusurkar R, Williams G. How self-determination theory can assist our understanding of the teaching and learning processes in medical education. AMEE Guide No. 59. Medical Teacher 2011;33:961-73.

10 Guckian J, Spencer J. \#SixSecondStudying: the rise and fall of Vine in MedEd. The Clinical Teacher 2018;16:164-6.

Address for correspondence: Dr Jonathan Guckian, Medical Education Department, St James' Hospital, Beckett Street, Leeds LS9 7TF, UK.

Email: jonathan.guckian@outlook.com

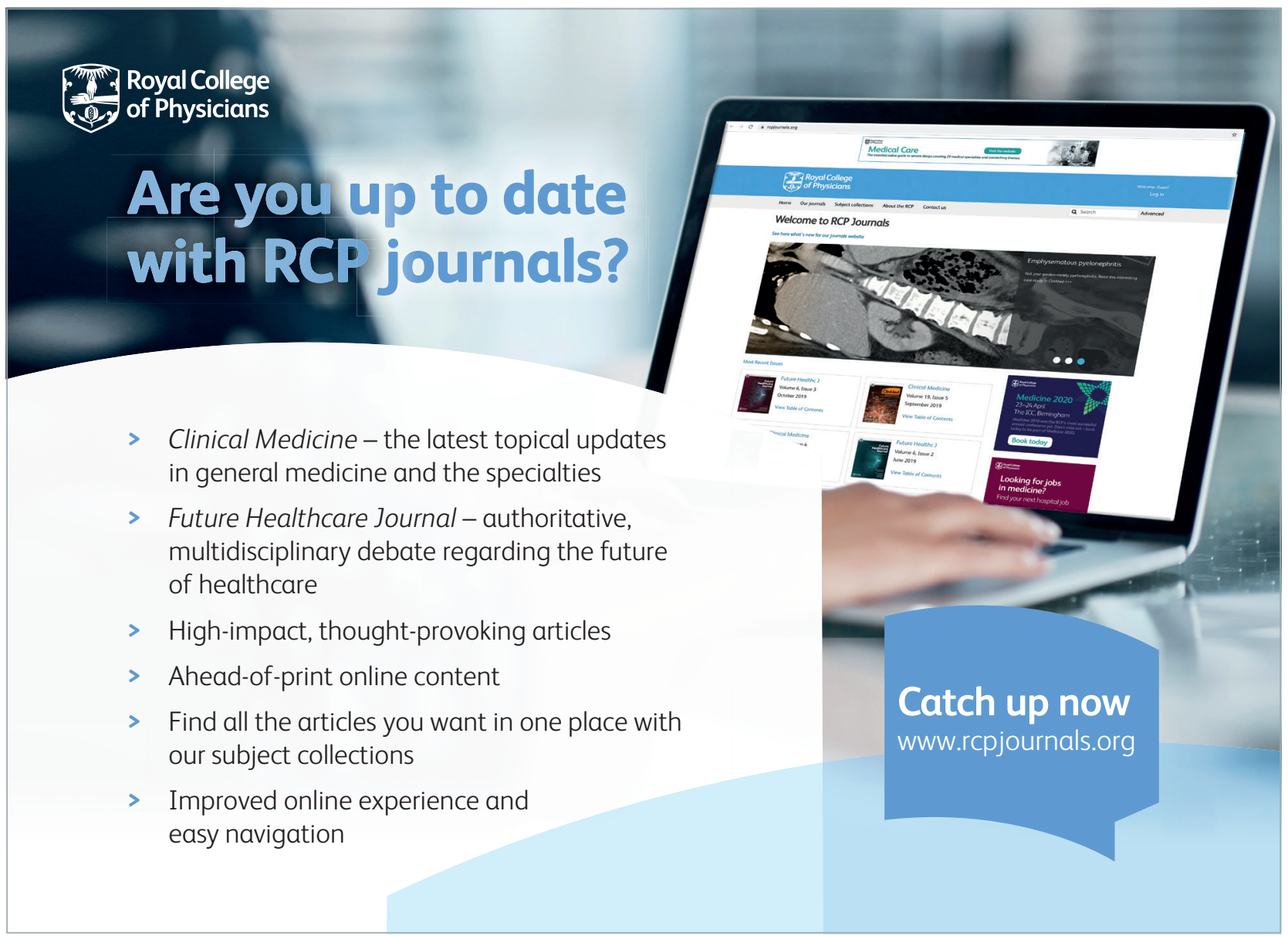

